Article

\title{
Anti-Angiogenesis Effect of Biogenic Silver Nanoparticles Synthesized Using Saliva officinalis on Chick Chorioalantoic Membrane (CAM)
}

\author{
Javad Baharara ${ }^{1,2}$, Farideh Namvar ${ }^{1,3, *}$, Marzieh Mousavi ${ }^{1}$, Tayebe Ramezani ${ }^{4}$ and \\ Rosfarizan Mohamad ${ }^{3,5}$
}

1 Research Center for Animal Development Applied Biology, Mashhad Branch, Islamic Azad University, Mashhad 917568, Iran; E-Mails: baharara@yahoo.com (J.B.);

m_moosavi_k@yahoo.com (M.M.)

2 Department of Biology, Mashhad Branch, Islamic Azad University, Mashhad 917568, Iran

3 Institute of Tropical Forestry and Forest Products (INTROP), Universiti Putra Malaysia, UPM Serdang, Selangor 43400, Malaysia; E-Mail: farizanmohd@gmail.com

4 Faculty of Biological Sciences, Kharazmi University, Tehran 14911, Iran; E-Mail: tayeberamezani@gmail.com

5 Department of Bioprocess Technology, Faculty of Biotechnology and Biomolecular Sciences, Universiti Putra Malaysia, UPM Serdang, Selangor 43400, Malaysia

* Author to whom correspondence should be addressed; E-Mail: farideh.namvar@putra.upm.edu.my; Tel.: +60-17-399-6005; Fax: +60-389-466-916.

Received: 1 June 2014; in revised form: 18 July 2014 / Accepted: 26 July 2014 /

Published: 1 September 2014

\begin{abstract}
Angiogenesis, which is required for physiological events, plays a crucial role in several pathological conditions, such as tumor growth and metastasis. The use of plant extracts is a cost effective and eco-friendly way to synthesize nanoparticles. In the present study, we investigated the anti-angiogenesis properties of silver nanoparticles synthesized using Saliva officinalis extract on chick chorioalantoic membrane. The production of nanoparticles was confirmed by the color change from yellow to brown observed after approximately $3 \mathrm{~h}$ at $37{ }^{\circ} \mathrm{C}$. Then, the nanoparticles were characterized by UV-visible spectroscopy, FTIR, and TEM. The UV-visible spectroscopy results showed that the surface plasmon resonance band for AgNPs was around $430 \mathrm{~nm}$. The intensity of the AgNP-specific absorption peak improved with an increase of $0.5 \mathrm{~mL}$ of extract into $10 \mathrm{~mL}$ of $\mathrm{AgNO}_{3}$ (2.5 mM). The FTIR results showed good interaction between the plant extracts and AgNPs. The TEM images of the samples revealed that the NPs varied in morphology
\end{abstract}


and size from 1 to $40 \mathrm{~nm}$; the average was recorded at $16.5 \pm 1.2 \mathrm{~nm}$. Forty Ross fertilized eggs were divided into four groups; the control and three experimental groups. On the 8th day, gelatin sponges containing albumin were placed on the chorioalantoic membrane and soaked with different concentrations of NPs. On the 12th day, all the cases were photographed using a photostereomicroscope. The number and the lengths of the vessels were measured using Image $J$ software. The crown rump (CR) and weight of the embryo were also recorded. Then the hemoglobin content was measured using Drabkin's reagent kit for quantification of the blood vessel formation. According to the data analysis, the number and length of the blood vessels, as well as the CR and weight of the embryos reduced significantly compared to the control $(p<0.05)$, dose dependently. The total hemoglobin was quantified as an indicator of the blood vessel formation. The hemoglobin content in the treated samples with AgNPs decreased, which showed its inhibitory effect on angiogenesis.

Keywords: angiogenesis; AgNPs; chorioalantoic membrane; Saliva officinalis

\section{Introduction}

The definition of tumor angiogenesis has been linked with Folkman's hypothesis about the growth of solid tumors as a result of blood vessel development [1]. Angiogenesis, which is the formation of new blood vessels from pre-existing ones, is regulated by the balance of many stimulating and inhibiting factors. While physiological angiogenesis is under strike control, the disruption of this control causes the proliferation of a network of blood vessels penetrating into cancerous growth [2]. Sage belongs to the genus Salvia of the Labiaceae family, which comprises about 900 plant species [3]. The plant is reported to have multiple pharmacological effects, including antibacterial, antiviral, anti-inflammatory, fungistatic, antimutagenic, anticancer and antioxidative effects [4-6]. The leaves of Salvia officinalis possess some therapeutic effects due to the presence of mainly flavonoids; phenolic compounds such as carnosic, rosmarinic, caffeic and salvianolic acids and other phenolic structure-based compounds that are especially found in the alcohol-soluble fraction [7]. The application of nanoparticle materials is an emerging area of nanotechnology [8]. Metals, such as platinum, silver and gold are commonly used for the synthesis of nanoparticles by chemical and biological methods [9]. However, most of the synthetic methods reported to date rely on organic solvents, such as $\mathrm{N}, \mathrm{N}$-dimethylformamide and toxic reducing agents like sodium borohydride, which result in serious environmental issues for industrial production [10]. The green synthesis of nanoparticles offers numerous benefits of eco-friendliness and compatibility for pharmaceutical and biomedical applications as they do not use toxic chemicals in the synthesis protocols [11-13]. AgNPs have obvious therapeutic potential in treating a variety of diseases, including retinal neovascularization, and acquired immunodeficiency syndrome [14]. Recently, there has been increased study on anti-angiogenesis and the anti-cancer effects of silver nanoparticles [15]. These findings confirm the antitumor properties of AgNPs, and indicate that these might be a cost-effective approach for the treatment of cancer cells [16]. Since there are no reports on the use of Saliva officinalis to synthesize AgNPs, and, also, in 
respect of the antioxidant compounds and widespread growth of Saliva officinalis, this plant may be considered as a potential agent for the eco-friendly synthesis of silver nanoparticles. Thus, in the present study, we decided to synthesize green AgNPs using Saliva officinalis extract and characterize it, to investigate its anti-angiogenesis properties.

\section{Results and Discussion}

\subsection{Synthesis of Ag-Nanoparticles and Characterized}

$\mathrm{AgNO}_{3}$ solution without the aerial plant part extract and the extract without $\mathrm{AgNO}_{3}$ did not show any change in color (Figure 1). Figure 2 provides an overview of the size range and distribution of the AgNPs, and shows spherical and pentagonal shapes with a size range of 1 to $40 \mathrm{~nm}$, with the average being recorded at $16.5 \pm 1.2 \mathrm{~nm}$. From this figure, it is clear that the frequency peak comes at approximately $10-20 \mathrm{~nm}$, and particle sizes ranging from 1 to $30 \mathrm{~nm}$ accounts for about $95 \%$ of the total particles observed.

The absorption spectrum of the yellowish-brown silver nanoparticle solution prepared with the proposed method showed a surface plasmon absorption band with a maximum of $430 \mathrm{~nm}$ (Figure 3), indicating the presence of spherical Ag nanoparticles. FTIR spectroscopy was used to identify the functional groups of the active components based on the peak value in the region of infrared radiation (Figure 4).

The DLS results revealed that the average size of the silver nanoparticles was $21 \mathrm{~nm}$. The presence of silver nanoparticles was further confirmed using EDX spectrometry, which confirmed the presence of silver. Metallic silver nanocrystals generally show a typical absorption peak at approximately $2.523 \mathrm{keV}$ due to surface plasmon resonance, which increases the confidence that silver has been correctly identified (Figure 5).

Figure 1. Visual appearance of vials containing the $S$. Officinalis extract and $\mathrm{AgNO}_{3}$ solution after different reaction times: (a) $\mathrm{AgNO}_{3} 5 \mathrm{mM}$, (b) Aerial part extract $S$. officinalis, (c) $\mathrm{AgNO}_{3}$.

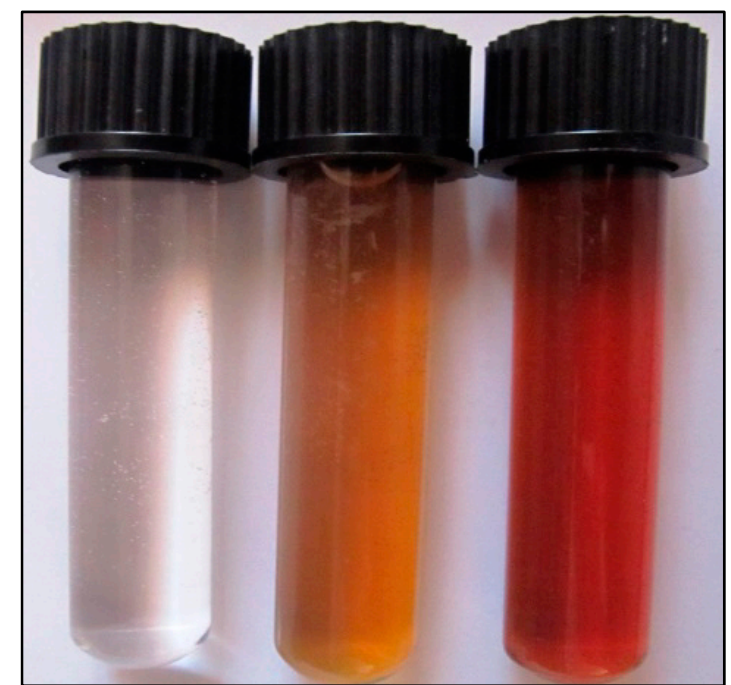

(a) (b)

(c) 
Figure 2. Transmission electron microscope images of the green synthesized silver NPs.

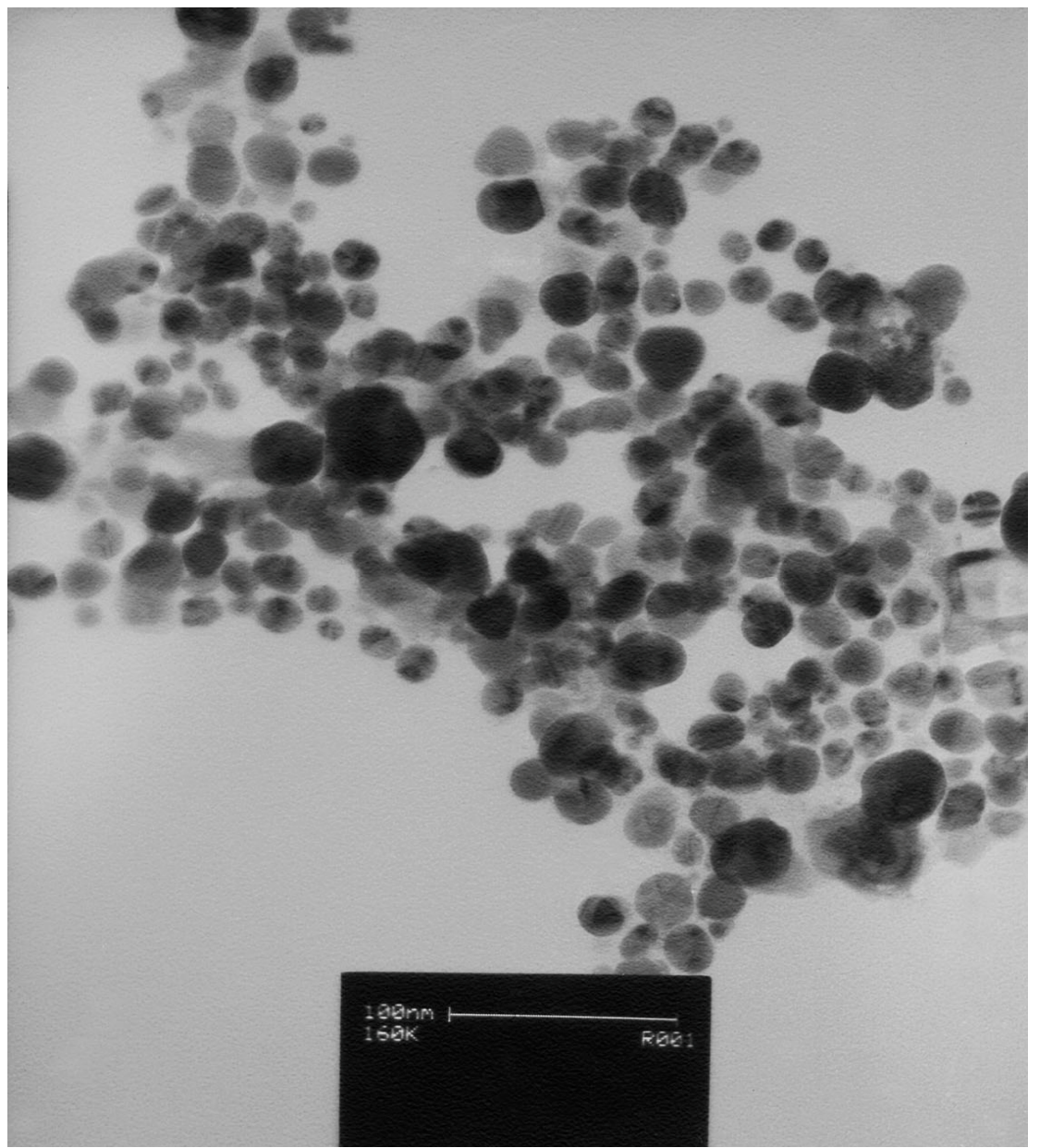

Figure 3. UV spectrophotometry of the green synthesized silver NPs.

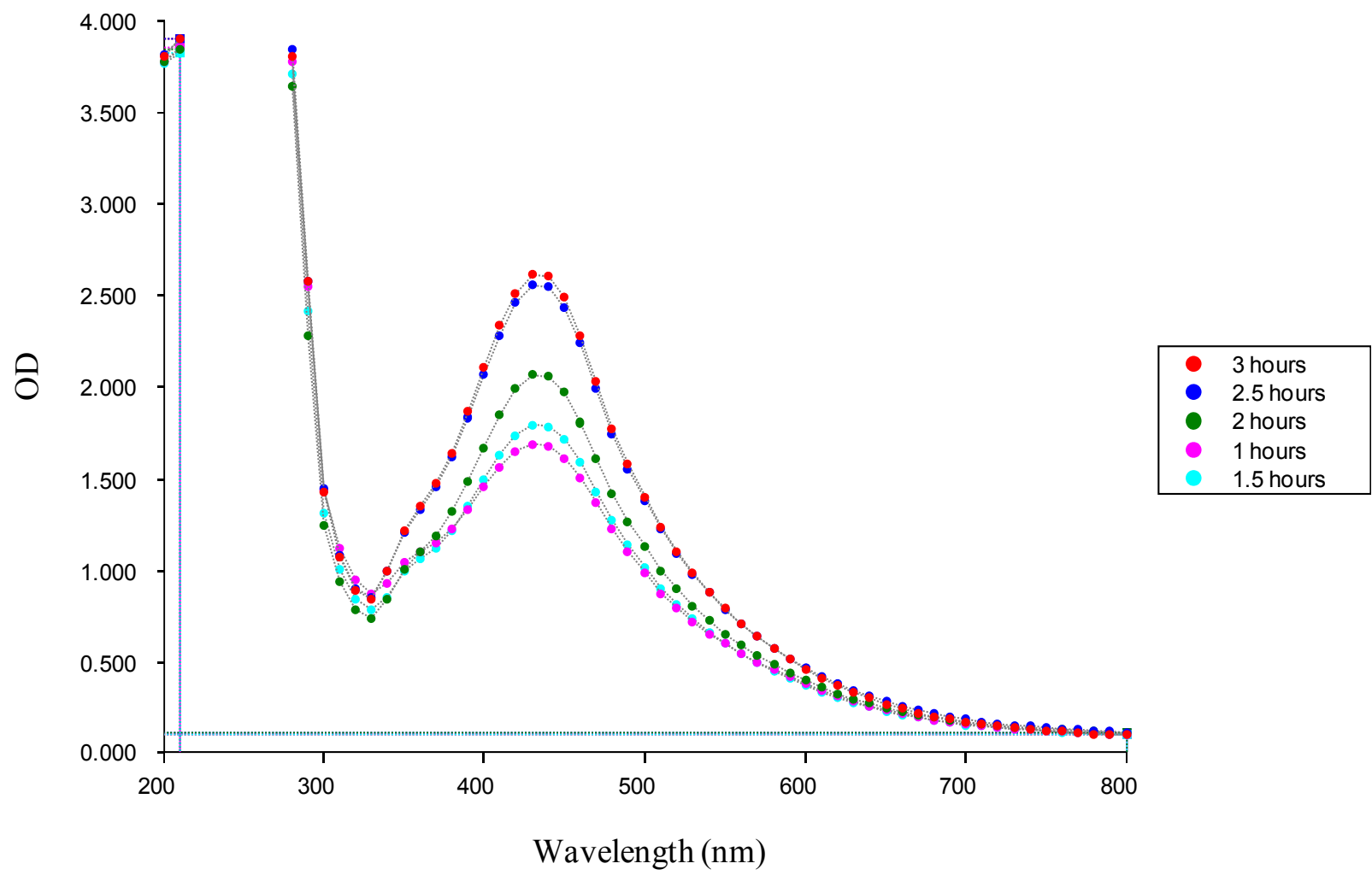


Figure 4. FTIR spectra of Saliva officinalis extract.

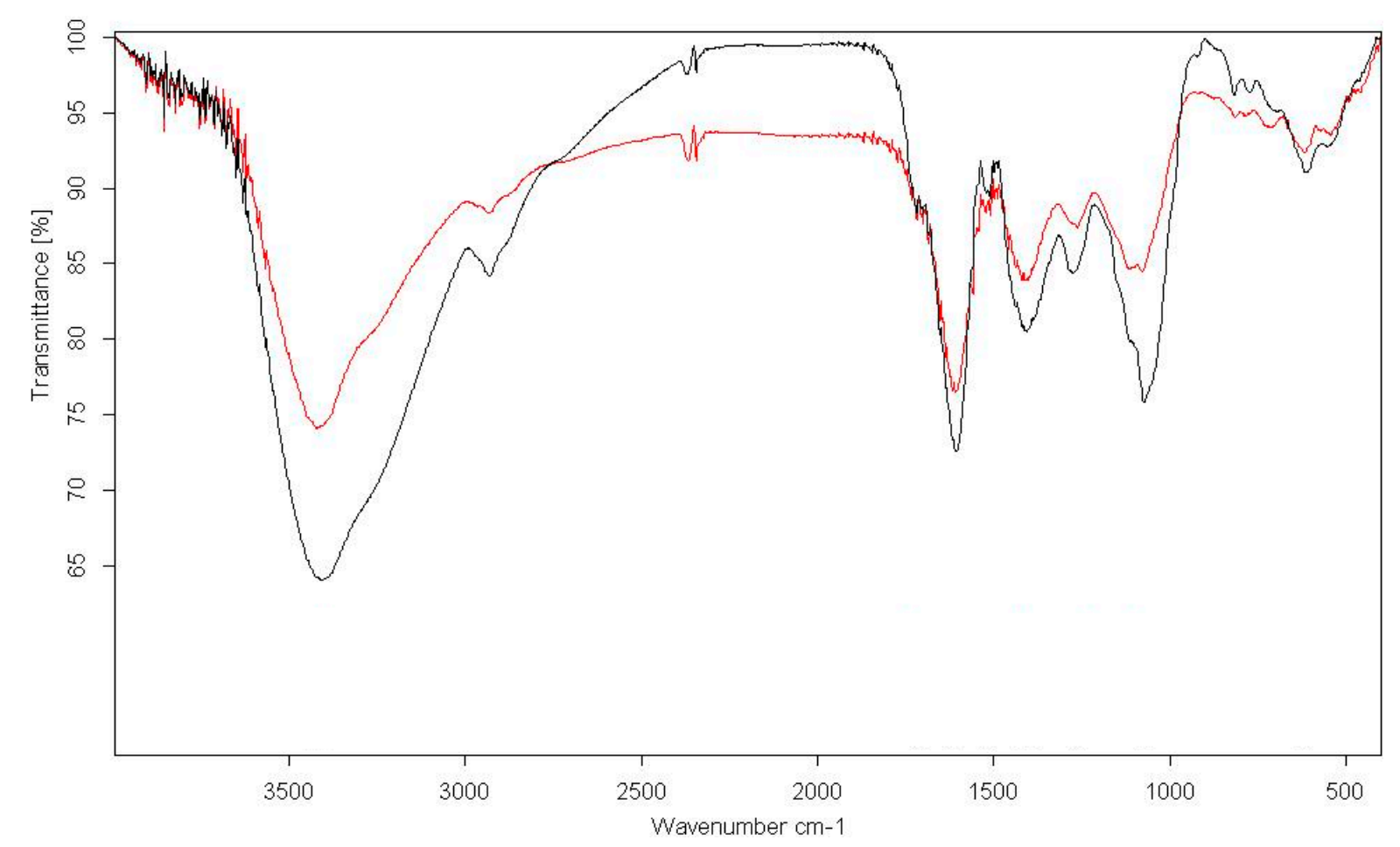

Figure 5. The particle size distribution of the synthesized silver nanoparticles.

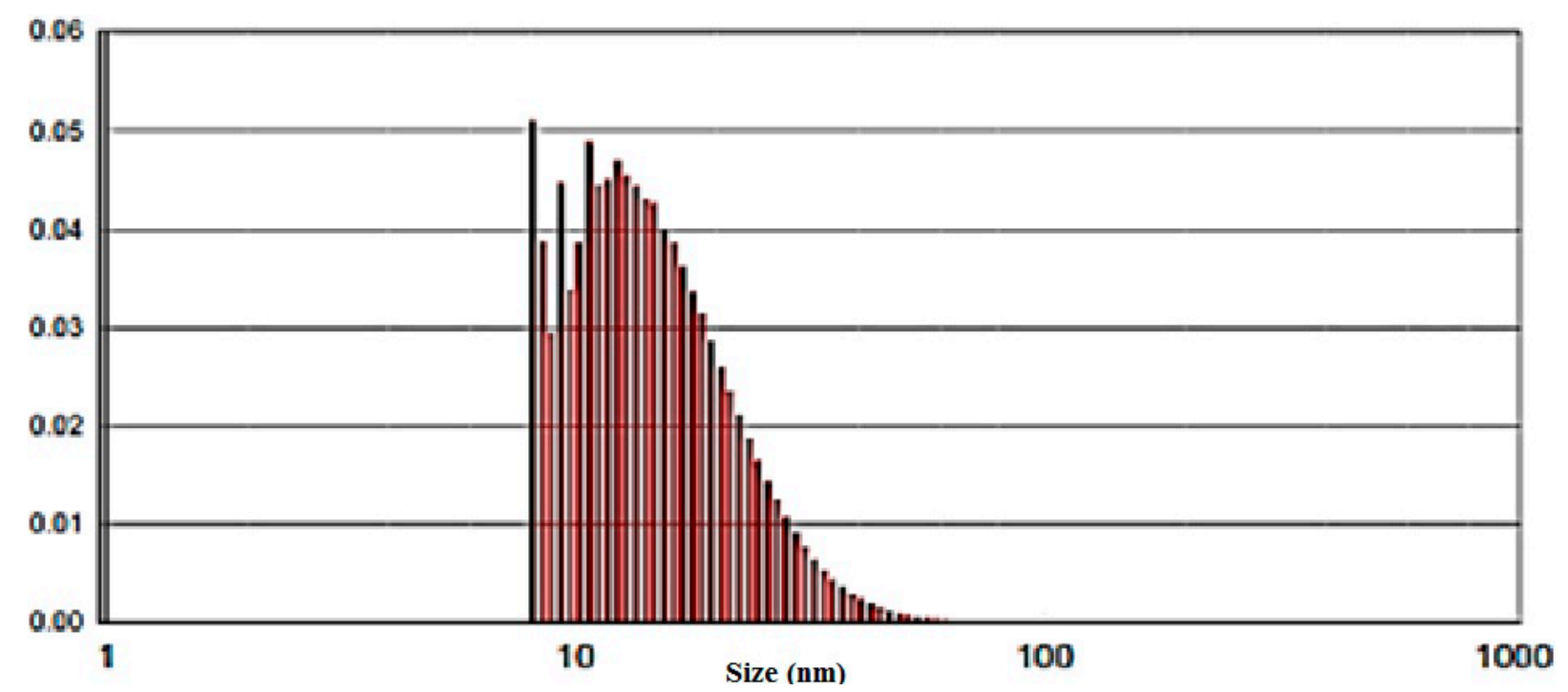

\subsection{Anti-Angiogenic Effect}

The chorioallantoic membrane (CAM) assay has been proved as a reliable in vivo model to study angiogenesis and many inhibitors and stimulators of angiogenesis have been examined by this common method [17]. Comparing the mean of the number (42.32 \pm 1.2$)$ and length $(29.72 \pm 1.6 \mathrm{~mm})$ of the control group with the treated samples with AgNPs at a concentration of $50 \mu \mathrm{g} / \mathrm{mL}:(40.09 \pm 0.7)$, $(24.36 \pm 2.3 \mathrm{~mm}) ; 100 \mu \mathrm{g} / \mathrm{mL}:(37.5 \pm 3.1),(19.85 \pm 3.6 \mathrm{~mm})$ and $200 \mu \mathrm{g} / \mathrm{mL}:(33.14 \pm 2.2),(17.14 \pm 1.7 \mathrm{~mm})$ showed a significant decrease $(p<0.05)$. According to data analysis, the samples treated with AgNPs showed a dose dependent decrease, which is shown in Figure 6. 
Figure 6. Average of number and length of blood vessels $(* p<0.05, * * p<0.001)$.
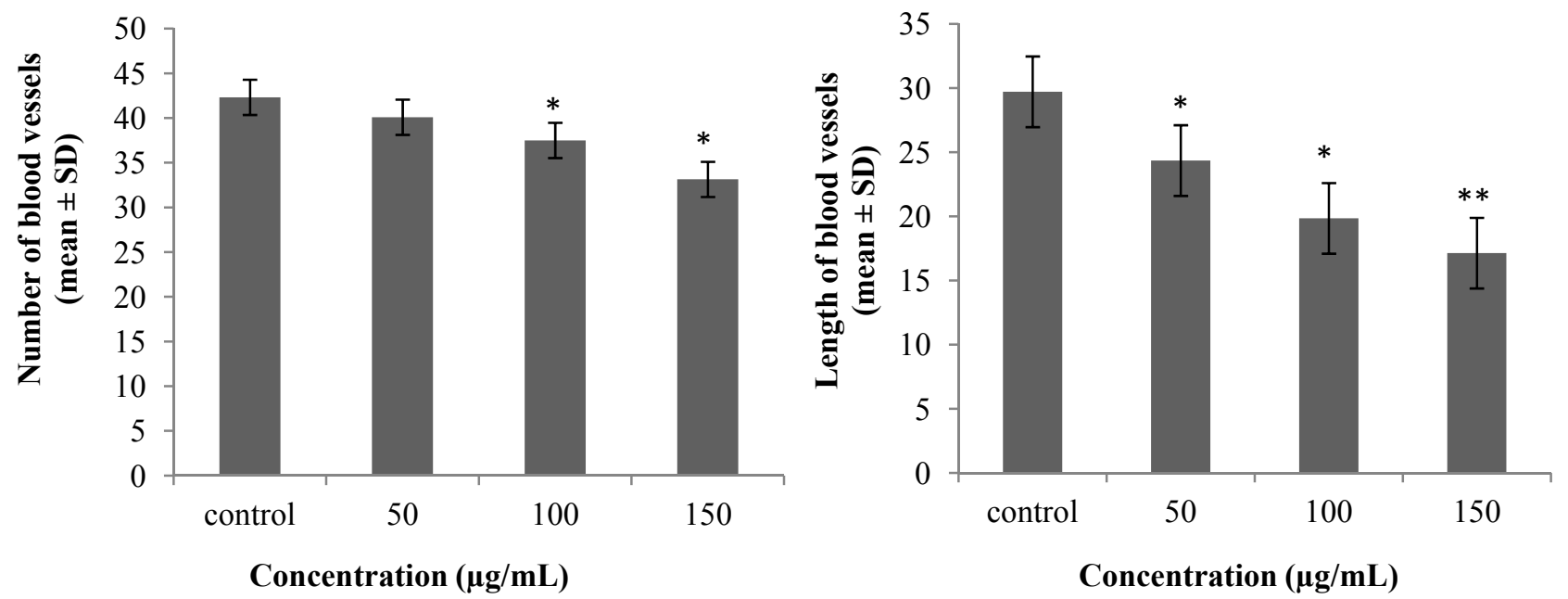

\subsection{Morphometric Analysis}

It is necessary to have knowledge about chick embryo development after treatment with different concentrations of AgNPs. Therefore, both the CR and weight of the treated samples were measured carefully to observe any probability of contamination (Figure 7). The CR $(4.3 \pm 0.2 \mathrm{~mm})$ and weight $(3.49 \pm 0.8 \mathrm{~g})$ of the control group, which were compared with the AgNPs treated samples at concentrations of $50 \mu \mathrm{g} / \mathrm{mL}:(4.1 \pm 0.4 \mathrm{~mm}),(3.27 \pm 0.5 \mathrm{~g}) 100 \mu \mathrm{g} / \mathrm{mL}:(3.7 \pm 0.7 \mathrm{~mm}),(3.15 \pm 0.1 \mathrm{~g})$ and $200 \mu \mathrm{g} / \mathrm{mL}(3.5 \pm 0.3 \mathrm{~mm}),(2.58 \pm 0.8 \mathrm{~g})$, revealed a noticeable dose dependent fall (Figure 8). Data analysis did not show any specific morphological changes, and development of the chick embryo was not affected by morphometric treatment of AgNPs.

Figure 7. Average of CR chick embryo.

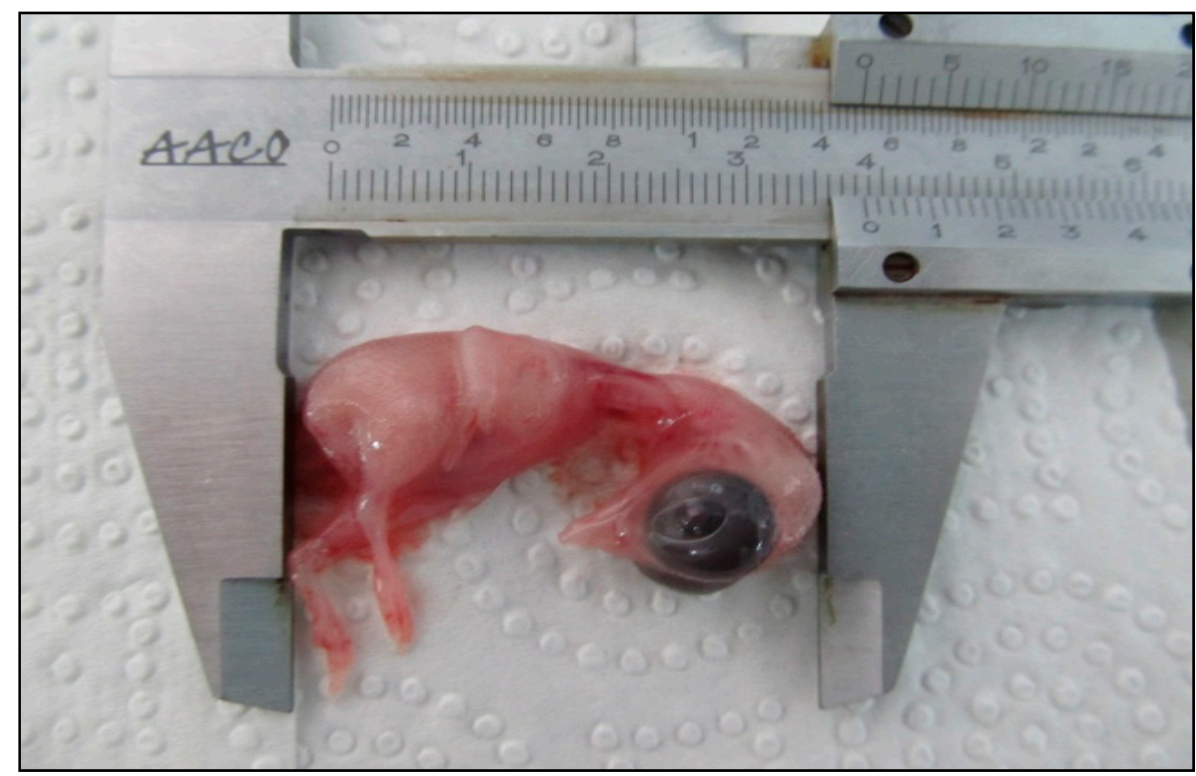


Figure 8. Average of CR and weight of chick embryo $(* p<0.05)$.
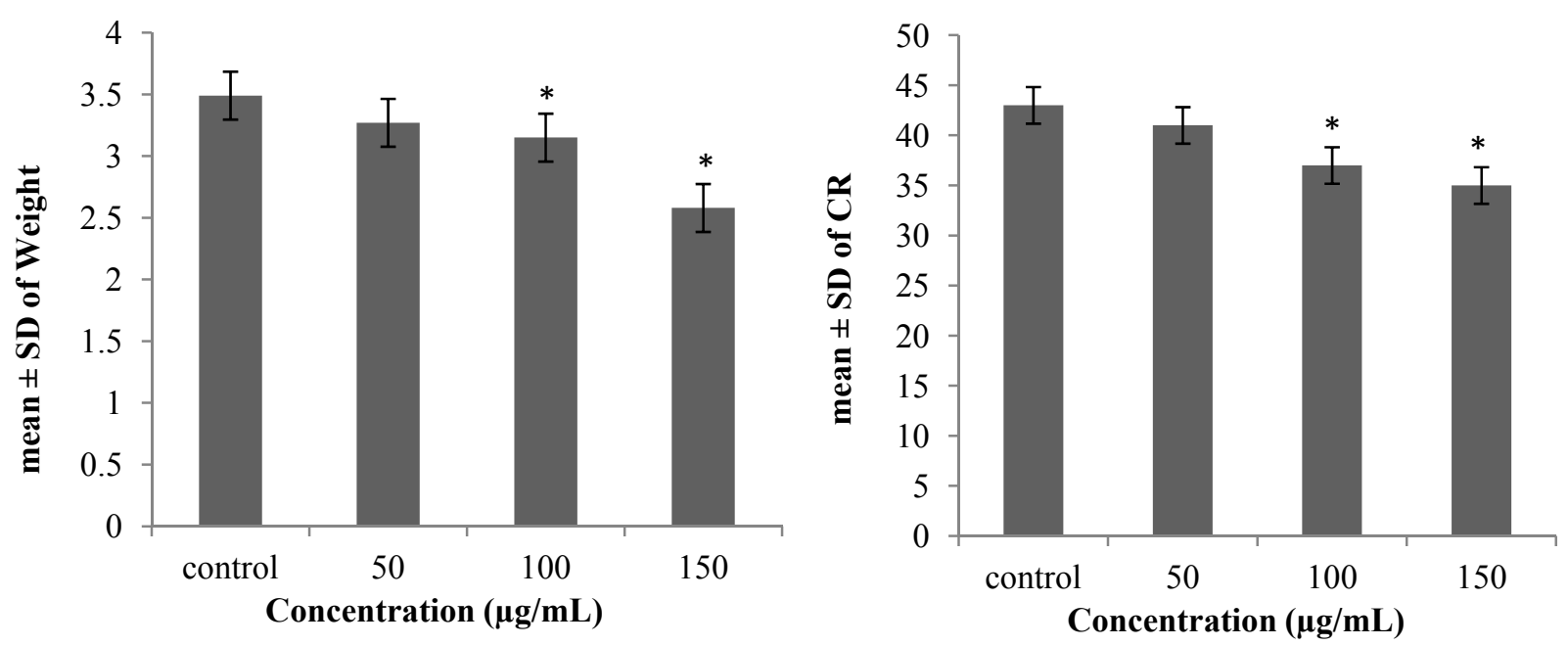

\subsection{Measurement of Hemoglobin}

The hemoglobin was measured for the quantification of blood vessel formation, using the Drabkin method $[18,19]$ as a measure of vessel density. This assay was used previously in other research and revealed great results that confirmed and proved the validity of the in vivo CAM assay [20]. The data analysis of the Drabkin test is revealed in Figure 9.

Figure 9. $\mathrm{Hg}$ absorbance (left) image of CAM with gelatin sponge (right). ${ }^{*} p<0.05,{ }^{* *} p<0.001$.
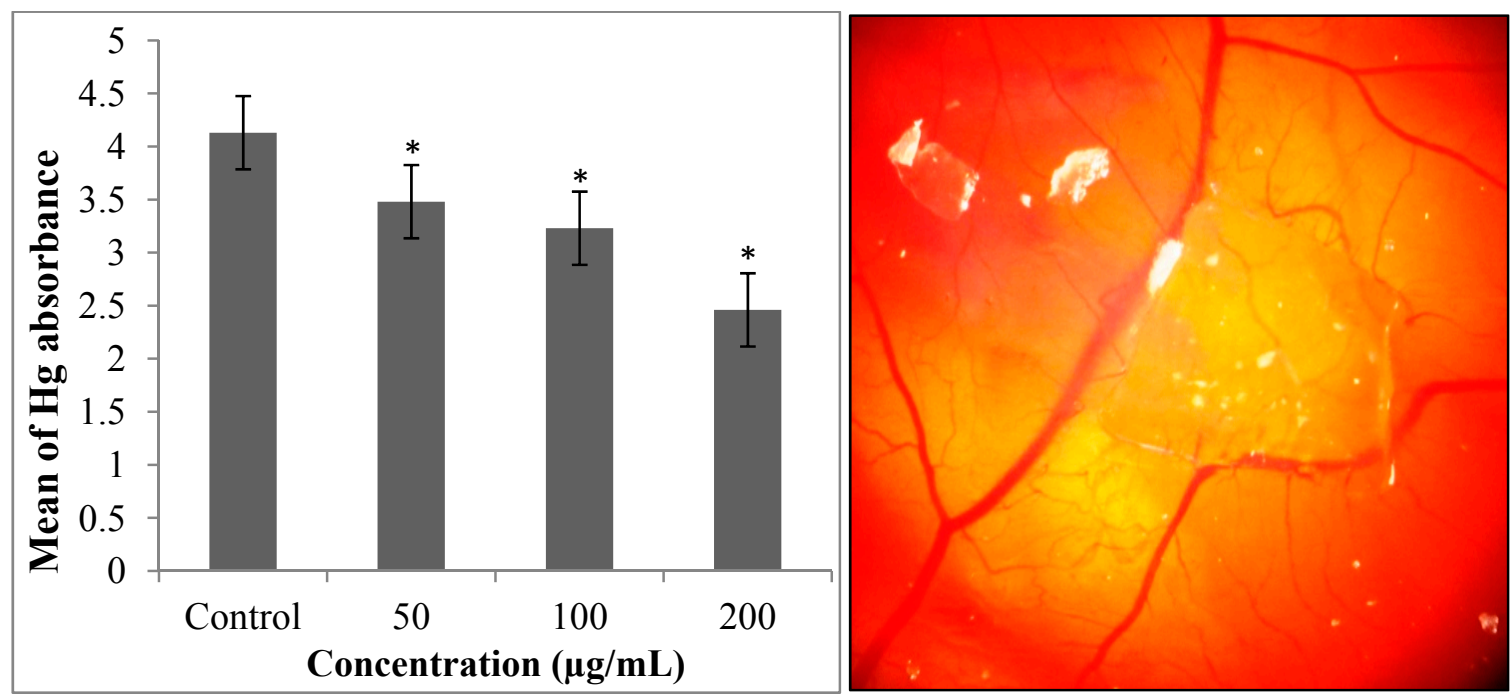

Another study also showed that silver nanoparticles led to subtle obstructive effects in the microcirculation of the chick embryo CAM [15]. These effects occurred without loss of embryo viability and were associated with the partial preservation of the capillary diameters and connectivity [21]. This particle cloud inhibited the vascular endothelial growth factor (VEGF) - induced cell proliferation, migration, and capillary-like tube formation of bovine retinal endothelial cells like PEDF (23). In addition, Ag-NPs effectively inhibited the formation of new blood micro vessels induced by VEGF. Similar studies have confirmed their inhibitory effect on vascular permeability induced by VEGF, interleukin (IL)-1 $\beta$, in retinal endothelial cells [22,23]. 


\section{Experimental Section}

\subsection{Reagents}

Silver nitrate was purchased from Merck (Darmstadt, Germany) and used as received. Distilled deionized water was used to prepare all the needed solutions. Saliva officinalis were obtained from a local source (Mashhad-Iran). The fertilized eggs were purchased from Toos Company (Mashhad, Iran) and the Drabkin kit from Zistchem (Tehran, Iran).

\subsection{Synthesis and Characterization of AgNPS}

We previously reported a green method for the synthesis of silver nanoparticles using Saliva officinalis. Briefly, the extract was prepared by taking dried aerial plant parts $(5 \mathrm{~g})$ in distilled water $(100 \mathrm{~mL})$ and thoroughly boiling the mixture for $10 \mathrm{~min}$. For the preparation of silver nanoparticles, an aliquot $(0.5 \mathrm{~mL})$ of the prepared extract of Saliva officinalis was typically added to $2.5 \mathrm{mM}$ aqueous silver nitrate solution $(10 \mathrm{~mL})$ and kept at $37{ }^{\circ} \mathrm{C}$ until the color of the solution slowly turned from yellow to dark brown, which clearly indicated the reduction of $\mathrm{Ag}^{+}$ions and formation of nanoparticles. The synthesis of AgNPs was characterized using a transmission electron microscope (Hitachi, Tokyo, Japan), DLS (Cordovan, Vaso particle, France), FT-IR (Perkin Elmer, Walthman, MA, USA) and EDX (XL 30; Philips, Eindhoven, The Netherlands).

\subsection{Angiogenesis Assay}

Forty Ross fertilized eggs were randomly divided into four groups - the control group, and experimental groups 1,2 and 3 (treated with concentrations of 50, 100 and 200 micrograms per milliliter AgNPs), and then incubated at $38{ }^{\circ} \mathrm{C}$ and $55 \%-65 \%$ humidity with automatic rotation in the incubation system. On day 2 of the incubation, a window was opened in the eggs in sterile condition, which was prepared using a laminar flow hood (Telstar, Madrid, Spain), part of the shell was removed and a small window opened, which was covered by sterile paraffin and lamellas (Iran Fara, Tehran, Iran). Then the eggs were transferred to an incubator and rotated manually twice a day for normal development of the embryos. On day 8 of incubation, a gelatin sponge containing gelatin in normal saline, and albumin with $200 \mu \mathrm{L}$ of penicillin and streptomycin was put on the chorioalantoic membrane and the experimental groups were soaked with $10 \mu \mathrm{L}$ of AgNPs and returned to the incubator. On the 12th day of incubation, all the cases were photographed using a research photostereomicroscope (Ziess, Munich, Germany). The variables include the number and length of blood vessels that for all the samples were measured around the gelatin sponge using the Image $\mathrm{J}$ software. The length and weight of the samples were recorded as morphometric traits to investigate their normal development or any disorder.

\section{Conclusions}

A critical need in the field of nanotechnology is the development of eco-friendly and reliable methods for the synthesis of nanoparticles. Here, we have reported a simple biological and low-cost approach for the preparation of stable silver nanoparticles by the reduction of silver nitrate solution 
with a bioreduction method using Saliva officinalis aqueous extract as the reducing agent. The characteristics of the obtained silver nanoparticles were studied using the UV-Vis, FTIR, EDX and TEM techniques. The results confirmed the reduction of silver nitrate to silver nanoparticles with high stability and without any impurity. Comparison of the experimental results showed that the average size of the synthesized silver nanoparticles was about $16.5 \mathrm{~nm}$. According to the data analysis, biologically synthesized silver nanoparticles could be of immense use in medicine and could also be considered as a promising chemotherapeutic agent in cancer treatment by exhibiting anti-angiogenic properties. The CAM structure, after treatment with toxic dosages of nanoparticles appeared to be clustered with a few cellular extensions and vessel formation, and cell-spreading patterns were restricted compared to the control groups. This could be due to disturbances in the cytoskeletal functions as a consequence of the nanoparticle treatment. Lower doses were not destroyed in vascular organization. In the present study, it was demonstrated that in the CAM model, silver nanoparticles have dose-dependent cytotoxic effects on endothelial cells and inhibited blood vessel formation.

\section{Acknowledgments}

This work was financially supported by the Research Center for Animal Development Applied Biology, Islamic Azad University Mashhad branch. The authors are sincerely thankful and grateful to the colleagues in Research Center for Animal Development for their help, assistance and kind support.

\section{Author Contributions}

Javad Baharara designed and supervised the experiments, analyzed the data and wrote the paper; Farideh Namvar designed and analyzed the data and wrote the paper; Tayebe Ramezani performed experiments, analyzed data and wrote the paper; Marzieh Mousavi performed experiments, analyzed data and wrote the paper; Rosfarizan Mohamad designed, analyzed data and wrote and edited the paper.

\section{Conflicts of Interest}

The authors declare no conflict of interests.

\section{References}

1. Bhat, T.A.; Singh, R.P. Tumor angiogenesis - a potential target in cancer chemoprevention. Food Chem. Toxicol. 2008, 46, 1334-1345.

2. Otrock, Z.K.; Hatoum, H.A.; Musallam, K.M.; Awada, A.H.; Shamseddine, A.I. Is VEGF a predictive biomarker to anti-angiogenic therapy? Crit. Rev. Oncol. Hematol. 2011, 79, 103-111.

3. Abu-Darwish, M.S.; Cabral, C.; Ferreira, I.V.; Gonçalves, M.J.; Cavaleiro, C.; Cruz, M.T.; Al-bdour, T.H.; Salgueiro, L. Essential oil of common sage (Salvia officinalis L.) from Jordan: Assessment of safety in mammalian cells and its antifungal and anti-inflammatory potential. Biomed Res. Int. 2013, 2013, 538940.

4. Xavier, C.; Lima, C.; Fernandes-Ferreira M.; Pereira-Wilson, C. Salvia fruticosa, Salvia officinalis, and rosmarinic acid induce apoptosis and inhibit proliferation of human colorectal cell lines: The role in MAPK/ERK pathway. Nutr. Cancer 2009, 61, 564-571. 
5. Khalil, W.K.B.; Abdu, F.; Biology, C. Effects of Salvia officinalis extract and its nano-encapsulated form on methylmercury induced neurotoxic-stress in male rats department of biological science, faculty of science. World Appl. Sci. J. 2013, 24, 826-837.

6. Namvar, F.; Suhaila, M.; Fard, S.G.; Behravan, J. Polyphenol-rich seaweed (Eucheuma cottonii) extract suppresses breast tumour via hormone modulation and apoptosis induction. Food Chem. 2012, 130, 376-382.

7. Lima, C.F.; Carvalho, F.; Fernandes, E.; Bastos, M.L.; Santos-Gomes, P.C.; Fernandes-Ferreira, M.; Pereira-Wilson, C. Evaluation of toxic/protective effects of the essential oil of Salvia officinalis on freshly isolated rat hepatocytes. Toxicol. In Vitro 2004, 18, 457-465.

8. Parashar, V.; Parashar, R.; Sharma, B.; Pandey, A.C. Parthenium leaf extract mediated synthesis of silver nanoparticles: A novel approach towards weed utilization. Dig. J. Nanomater. Bios. 2009, 4, 45-50.

9. Ranjan, P.; Das, M.; Kumar, M.; Anbarasi, P.; Sindhu, S.; Sagadevan, E.; Arumugam, P. Green synthesis and characterization of silver nanoparticles from Nigella sativa and its application against UTI causing bacteria. J. Acad. Ind. Res. 2013, 2, 45-49.

10. Pandian M.; Marimuthu, R.; Natesan, G.; Rajagopal, R.E.; Justin, J.S.; Mohideen, A.J.A.H. Development of biogenic silver nano particle from Pelargonium graveolens leaf extract and their antibacterial activity. Am. J. Nanosci. Nanotechnol. 2013, 1, 57-64.

11. Baharara, J.; Namvar, F.; Ramezani, T.; Hosseini, N.; Mohamad, R. Green synthesis of silver nanoparticles using achillea biebersteinii flower extract and its anti-angiogenic properties in the rat aortic ring model. Molecules 2014, 19, 4624-4634.

12. Dubey, M.; Bhadauria, S.; Kushwah, B. Green synthesis of nanosilver particles from extract of Eucalyptus hybrida (safeda) leaf. Dig. J. Nanomater. Bios. 2009, 4, 537-543.

13. Mahdavi, M.; Namvar, F.; Ahmad, M.B.; Mohamad, R. Green biosynthesis and characterization of magnetic iron oxide $\left(\mathrm{Fe}_{3} \mathrm{O}_{4}\right)$ nanoparticles using seaweed (Sargassum muticum) aqueous extract. Molecules 2013, 18, 5954-5964.

14. Sharma, V.K.; Yngard, R.A.; Lin, Y. Silver nanoparticles: Green synthesis and their antimicrobial activities. Adv. Colloid Interface Sci. 2009, 145, 83-96.

15. Gurunathan, S.; Lee, K.-J.; Kalishwaralal, K.; Sheikpranbabu, S.; Vaidyanathan, R.; Eom, S.H. Antiangiogenic properties of silver nanoparticles. Biomaterials 2009, 30, 6341-6350.

16. Kumar, V.; Yadav, S. Plant-mediated synthesis of silver and gold nanoparticles and their applications. J. Chem. Technol. Biotechnol. 2009, 84, 151-157.

17. Ribatti, D. The chick embryo chorioallantoic membrane as an in vivo assay to study antiangiogenesis. Pharmaceuticals 2010, 3, 482-513.

18. Xavier, D.O.; Amaral, L.S.; Gomes, M.A.; Rocha, M.A.; Campos, P.R.; Cota, B.D.C.V; Tafuri, L.S.A.; Paiva, A.M.R.; Silva, J.H.; Andrade, S.P.; et al. Metformin inhibits inflammatory angiogenesis in a murine sponge model. Biomed. Pharmacother. 2010, 64, 220-225.

19. Araújo, F.A.; Rocha, M.A.; Mendes, J.B.; Andrade, S.P. Atorvastatin inhibits inflammatory angiogenesis in mice through down regulation of VEGF, TNF-alpha and TGF-beta1. Biomed. Pharmacother. 2010, 64, 29-34.

20. Viji, R.; Kumar, V.; Kiran, M.; Sudhakaran, P. Angiogenic response of endothelial cells to heparin-binding domain of fibronectin. Int. J. Biochem. Cell Biol. 2008, 40, 215-225. 
21. Wierzbicki, M.; Sawosz, E.; Grodzik, M.; Hotowy, A.; Prasek, M.; Jaworski, S.; Sawosz, F.; Chwalibog, A. Carbon nanoparticles downregulate expression of basic fibroblast growth factor in the heart during embryogenesis. Int. J. Nanomed. 2013, 8, 3427-3435.

22. Wierzbicki, M.; Sawosz, E.; Grodzik, M.; Prasek, M.; Jaworski, S.; Chwalibog, A. Comparison of anti-angiogenic properties of pristine carbon nanoparticles. Nanoscale Res. Lett. 2013, 8, 195.

23. Sheikpranbabu, S.; Kalishwaralal, K.; Venkataraman, D.; Eom, S.H.; Park, J.; Gurunathan, S. Silver nanoparticles inhibit VEGF-and IL-1beta-induced vascular permeability via Src dependent pathway in porcine retinal endothelial cells. J. Nanobiotechnol. 2009, 7, 8.

Sample Availability: Samples of the different experiments are available from the authors.

(C) 2014 by the authors; licensee MDPI, Basel, Switzerland. This article is an open access article distributed under the terms and conditions of the Creative Commons Attribution license (http://creativecommons.org/licenses/by/3.0/). 